Revue d'histoire de l'Amérique française

DQ REVUE D.HISTOIRE DE L'AMÉRIQUE FRANÇAISE

\title{
Le Québec contemporain vu par Paul-André Linteau, René Durocher, Jean-Claude Robert et François Ricard
}

\section{Bernard L. Vigod}

Volume 41, numéro 2, automne 1987

URI : https://id.erudit.org/iderudit/304553ar

DOI : https://doi.org/10.7202/304553ar

Aller au sommaire du numéro

Éditeur(s)

Institut d'histoire de l'Amérique française

ISSN

0035-2357 (imprimé)

1492-1383 (numérique)

Découvrir la revue

Citer cette note

Vigod, B. L. (1987). Le Québec contemporain vu par Paul-André Linteau, René Durocher, Jean-Claude Robert et François Ricard. Revue d'histoire de l'Amérique française, 41(2), 237-243. https://doi.org/10.7202/304553ar d'utilisation que vous pouvez consulter en ligne. 


\section{NOTE CRITIQUE}

\section{LE QUÉBEC CONTEMPORAIN VU PAR \\ PAUL-ANDRÉ LINTEAU, RENÉ DUROCHER, JEAN-CLAUDE ROBERT ET FRANÇOIS RICARD}

Tout commentaire critique à propos de cet ouvrage ${ }^{1}$ doit être fait en gardant à l'esprit que ce travail représente une réalisation extraordinaire. Il aborde à peu près toutes les facettes imaginables de l'évolution du Québec durant les cinquante dernières années et, même si ses auteurs s'en défendent, il peut être lu avec profit par quiconque s'intéresse au développement canadien. Plusieurs manuels d'histoire du Canada au 20e siècle présentent des faiblesses lorsqu'ils abordent l'histoire du Québec, et on pourrait même dire que Le Québec depuis 1930 se compare avantageusement à ceux-ci lorsqu'il s'agit d'écrire l'histoire du Canada.

L'ouvrage se divise chronologiquement en trois sections couvrant respectivement la crise et la Deuxième Guerre mondiale, la période duplessiste, et enfin les années depuis la Révolution tranquille. Selon une perspective structuraliste, les chapitres à l'intérieur de chacune des sections touchent des phénomènes sociaux, économiques, politiques et culturels. On devra examiner les trois sections séparément, puisque les sources utilisées par les auteurs et les perspectives que ceux-ci adoptent, diffèrent considérablement. Ceci concerne particulièrement la période commençant avec les années 60 , laquelle occupe presque la moitié des 726 pages. C'est à ce moment, en effet, que les auteurs sont confrontés aux débats et problèmes liés à l'écriture de l'histoire qui se fait encore et dont ils sont partie prenante.

La partie couvrant les années 1930-1945 se présente comme une suite logique à la dernière section du volume précédent ${ }^{2}$ qui portait sur la période de 1897 à 1929. La division des chapitres y est semblable, et le lecteur est particulièrement bien préparé à comprendre les effets de la crise sur l'économie du Québec. Cette section a de nombreuses

\footnotetext{
1 Paul-André Linteau, René Durocher, Jean-Claude Robert et François Ricard, Histoire du Québec contemporain: le Québec depuis 1930 (Montréal, Boréal Express, 1986), 739 p.

2 Paul-André Linteau, René Durocher et Jean-Claude Robert, Histoire du Québec contemporain, vol. 1: De la Confédération à la crise. Montréal, Boréal Express, 1979. 660 p.
} 
qualités. Elle campe solidement le Québec dans les contextes canadien et nord-américain, et cela, non seulement sur le plan économique mais aussi socialement et politiquement. En juxtaposant deux sous-périodes, elle décrit adroitement l'impact de la guerre. Cette partie démontre la maîtrise de l'important ensemble de recherches historiques sur cette période. Elle rend intelligible l'histoire culturelle des élites et du peuple à ceux, étudiants et professionnels, qui sont habituellement plus familiers avec l'histoire économique et politique. L'étude du nationalisme est un modèle d'objectivité: alors que les mouvements nationalistes de la période sont normalement les sujets d'ardentes polémiques, les auteurs présentent ici le nationalisme comme un courant idéologique parmi d'autres.

Comme toujours, on pourrait se plaindre que l'approche structuraliste oblige à un nombre considérable de répétitions, introduisant du même coup quantité de divisions artificielles entre des sujets pourtant apparentés. Je soupçonne toutefois que la séparation de la vie politique et des développements socio-économiques par les auteurs, résulte autant de leur sous-estimation du leadership politique du Québec que de leur méthode d'analyse. Plus surprenante encore est leur réticence à décrire de façon imagée la vie quotidienne des Québécois. Étant donné les intérêts des historiens québécois pour les expériences personnelles, la richesse des sources disponibles et les événements dramatiques amenés par la dépression et la guerre, les auteurs auraient certainement pu s'éloigner du portrait aride qui résulte de l'énumération de statistiques. Il est à souligner que les conséquences de ce mode d'exposition ne sont pas uniquement esthétiques: le texte ignore résolument l'impact psychologique de la crise sur les individus, un aspect pourtant bien développé par les historiens anglophones et essentiel à une compréhension globale de l'implantation de «l'État-providence» au Canada. Les auteurs font bien allusion aux attentes populaires lorsqu'ils mentionnent le gouvernement réformiste de Godbout, mais ils ne semblent pas en dégager toutes les significations. Il y a certainement des différences idéologiques majeures entre le Manifeste de Régina et le Programme de restauration sociale ou la plate-forme de l'Action libérale nationale, mais ils expriment tous, en pratique, le besoin de protéger la dignité de l'individu en lui assurant certaines formes de sécurité du revenu. Dans cette perspective, le vaste support accordé par le Québec aux libéraux fédéraux et à leurs visées centralisatrices après la guerre se comprend mieux: sur cette question, les Québécois participaient au consensus national. En fait, on peut critiquer les auteurs, de façon générale, pour ne pas avoir reconnu l'impact du Québec sur les politiques fédérales. Tout comme la popularité des mesures de sécurité sociale au Québec a encouragé les libéraux à agir après la guerre, la peur de la réaction du Québec a eu beaucoup à faire avec leur timidité sur les questions sociales tout au long de l'entre-deux-guerres. Incidemment, c'était Mackenzie 
King et non les gouvernements du Québec et de l'Ontario, qui fit rejeter une partie importante du «New Deal» de R. B. Bennett par les tribunaux. On peut aussi mentionner l'influence décisive du Québec sur la politique étrangère du Canada durant les années 1930 et sur l'organisation de l'effort de guerre. Les auteurs n'avaient pas besoin d'être éblouis par les représentants du Québec à Ottawa durant cette période pour reconnaître le pouvoir politique de l'opinion canadienne-française.

Pour la période de l'après-guerre, «A l'ombre de Duplessis», on retrouve encore de nombreux éléments intéressants. Cette partie décrit bien les changements profonds amenés par la prospérité, la croissance économique et les débuts de la révolution dans les communications. Implicitement, sinon explicitement, on y reprend les conclusions de la plupart des recherches spécialisées: beaucoup de choses bougeaient sous le vernis pétrifié de l'ancien régime. La Révolution tranquille, tout au moins au sens étroit (modernisation politique et réforme des institutions), représentait d'abord l'aboutissement d'un processus auquel Duplessis avait résisté. L'importance accordée par les auteurs aux aspirations et autres caractéristiques de la plupart des réformistes est une précaution bienvenue contre la tentation de projeter dans le passé la polarisation des années 60 et 70 entre libéraux et nationalistes. L'analyse brillante du développement urbain (et de celui des banlieues) témoigne de toute évidence de l'expertise d'un des auteurs; comme la plupart des historiens ne sont pas familiers avec ce sujet, il occupe souvent une position bien discrète en dépit de son importance critique dans la période d'après-guerre. Le récit de l'évolution culturelle du Québec pour cette période est à nouveau une agréable surprise, autant pour les professeurs que pour les élèves. Plus encore, ce récit fait quitter à la culture sa position marginale et l'amène au centre de la dynamique historique.

La plupart des faiblesses de cette partie devraient plutôt être appelées des désappointements. Des débats historiographiques intéressants existent à propos de la période duplessiste, mais les auteurs ne les développent pas explicitement. A l'opposé, le volume précédent foisonnait de discussions instructives sur l'historiographie. Puisque tous les éléments nécessaires à une telle discussion étaient présents, il aurait semblé bien naturel d'éclairer et de confronter les deux courants rivaux, «15 ans de réalisations» et «la grande noirceur», tout comme les diverses tentatives pour expliquer la popularité et la longévité du régime duplessiste. Au lieu de cela, nous voyons les auteurs reprendre certains mythes sans leur apporter de nouveaux supports: par exemple que Duplessis dirigeait à lui seul le gouvernement en l'absence de ministres compétents dans son cabinet, ou que Louis Saint-Laurent a trahi GeorgesÉmile Lapalme sur la question de l'impôt sur le revenu. Et puisque le terme «modernisation» revient fréquemment, on peut se demander 
pourquoi les auteurs ne précisent pas la façon dont McRoberts et Postgate utilisent la théorie de la modernisation pour expliquer les sources d'appui et d'opposition au régime duplessiste. Les défenseurs du nationalisme traditionnel sont identifiés (e.g. p. 325), mais leur comportement n'est pas expliqué de façon systématique.

La seconde déception majeure est la préférence tenace des auteurs pour des données statistiques et d'insipides généralisations. On se demande réellement en quoi les principes scientifiques seraient heurtés par un exposé qui tiendrait compte du drame et colorerait un peu les événements captivants et les personnages de la période. En abusant d'une approche struturaliste, les auteurs ont raté plusieurs occasions d'intéresser nos étudiants au passé. Victimes des bureaucraties du monde de l'éducation, élèves et professeurs mériteraient certainement d'en savoir plus au sujet du frère Untel.

Finalement, et même si ce compte rendu ne vise pas à énumérer des questions de détail, il y a quelques absences curieuses dans le chapitre consacré aux travailleurs. Un résumé des points d'affrontement dans les conflits de travail de la période ignore la question des revenus et bénéfices! Et on n'y fait pas référence aux changements de leadership ayant cours à l'intérieur de la CTCC, changements qui ont eu des effets sur sa situation. Il y $a$ des allusions à cet égard dans un chapitre ultérieur, ce qui illustre à nouveau les dangers de ce type de présentation.

La dernière tranche de l'ouvrage affronte le défi d'écrire l'histoire contemporaine. Même si les auteurs font montre d'une connaissance remarquablement détaillée de la période postérieure à 1960, et même si plusieurs chapitres sont très bien faits, la possibilité d'une synthèse s'éclipse avec la prolifération inexplicable des catégories. Il n'y a pas moins de 24 chapitres portant sur autant de thèmes particuliers; non seulement les chevauchements introduisent des répétitions fastidieuses, mais ils provoquent aussi la fragmentation de sujets majeurs qui auraient nécessité un traitement en profondeur. Par exemple, même si un chapitre est consacré au féminisme, on doit parcourir les chapitres sur la démographie, la société de consommation, les politiques sociales, les travailleurs, l'éducation et les partis politiques avant d'obtenir toutes les informations nécessaires à la compréhension du statut des femmes. Et il n'y a pas de discussion de la critique féministe du nationalisme québécois actuel, en dépit de l'importance de ce thème dans les travaux des historiennes - et cela, sans mentionner le phénomène des "Yvette». Bien que le problème des définitions soit discuté, le lecteur doit forger sa propre interprétation de la Révolution tranquille. Considérant l'abondante littérature écrite sur les années 60 et les positions très nettes qui s'en dégagent, on aurait pu s'attendre à une discussion historiographique importante. Alors que les auteurs pouvaient être en droit de décrire l'idéologie radicale comme une force marginale dans la société 
québécoise, le défi intellectuel posé à cette société par le mouvement marxiste mérite d'être considéré sérieusement.

Même si il n'y a pas de propagande, ni d'intention de provoquer une polémique, (au contraire, on pourrait plutôt dire que les auteurs cachent l'intensité de certains débats) on décèle des échos de la campagne référendaire à chaque fois que le gouvernement fédéral ou ses dirigeants sont mis en cause. Les réalisations des Canadiens français à Ottawa sont systématiquement ignorées, diminuées ou dépréciées: les premières tentatives et démarches pour obtenir une plus grande influence dans le gouvernement fédéral ne font pas partie de la Révolution tranquille (en fait, on laissera entendre plus tard qu'aucun progrès n'est survenu pour corriger la sous-représentation des francophones aux échelons supérieurs de la fonction publique); le bilinguisme au fédéral devient un facteur négatif, interférant avec l'affirmation de l'État du Québec; la diminution de l'importance numérique du Québec dans la Confédération est mentionnée sans qu'on ne fasse référence à l'influence croissante de celui-ci à l'intérieur du gouvernement fédéral; la participation de francophones aux travaux de la Commission royale d'enquête sur la condition de la femme au Canada n'est pas mentionnée; la discussion du «nationalisme canadien» chez les francophones québécois ignore tout à fait la dimension économique - la croyance que le système économique canadien administré par le gouvernement fédéral est viable et essentiel à la prospérité du Québec. Et sûrement, à moins de ne parler que de Pierre-Elliott Trudeau, c'est une exagération de dire que les francophones partisans du nationalisme canadien s'opposent au nationalisme québécois «sous toutes ses formes». Les auteurs eux-mêmes s'expriment différemment dans un autre contexte (p. 519 et 619$)$.

Lorsqu'est examiné l'impact du gouvernement fédéral sur l'économie, nous croyons revivre la «bataille des chiffres» qui a précédé le référendum - et aucun doute ne subsiste quant à la position des auteurs à cet égard. «On veut que l'intervention étatique provinciale permette de contrer les effets négatifs des politiques fédérales pour les francophones» et «la concentration de l'activité économique en Ontario... est favorisée par les politiques dites nationales dont les critères et les objectifs répondent d'abord à ses besoins», mais ces affirmations ne sont nulle part étayées par des documents. A nouveau sans preuve, il est dit que le gouvernement Trudeau, lorsqu'il entreprend de limiter la propriété des compagnies de transport par des agences des gouvernements provinciaux, représente «l'establishment canadien-anglais» contre «la montée des entreprises francophones». De la même façon, l'argent transféré au Québec par «Trudeau et ses ministres» à travers divers programmes et ententes ne parvient pas à contrebalancer des politiques (non spécifiées) qui favoriseraient la concentration de l'activité économique en Ontario. Bien sûr, ces remarques amuseraient, sinon outra- 
geraient les représentants de l'Atlantique ou des Prairies; ils apporteraient comme argument assez juste que le Québec et l'Ontario, ensemble, sont les bénéficiaires de la concentration économique à leurs dépens, et que la désignation de Montréal par Jean Marchand comme une région à «croissance lente» a effectivement empêché le ministère de l'Expansion économique régionale d'appuyer les tentatives des régions défavorisées pour attirer des investissements dans le secteur manufacturier. Ailleurs dans leur livre, les auteurs montrent leur connaissance du fait que le déplacement des bureaux-chefs vers l'ouest et que le taux de croissance généralement plus élevé de l'Ontario, ne résultent pas d'abord de politiques fédérales. D'un autre côté, ils refusent de reconnaître des facteurs politiques, particulièrement les controverses autour de la langue, pour expliquer la perte d'industries au Québec.

Le récit des événements politiques des années 80 est plongé dans le pessimisme. Il n'y a pas d'interprétation explicite des résultats du référendum, alors que le débat constitutionnel de 1981-1982 reçoit un traitement singulièrement bref. De toute façon, les auteurs laissent voir leurs réactions ailleurs. Même s'il reflète probablement le conservatisme et l'individualisme fort répandus dans les pays occidentaux, le calme idéologique qui a suivi le référendum témoigne aussi d'une «rupture» fatale - la dernière défaite - de la Révolution tranquille. Une fois que la Révolution tranquille est définie comme l'affirmation progressive de l'État du Québec par la collectivité francophone, cette conclusion devient un axiome. Mais il y a d'autres interprétations possibles, que les auteurs auraient pu présenter, même s'ils ne les partagent pas. Peut-être la Révolution tranquille fut-elle en fait un succès! Personne n'oserait contester aujourd'hui le principe de l'égalité linguistique dans les institutions fédérales, et des progrès dans la poursuite de cet objectif sont constamment observés malgré d'occasionnelles réactions agressives du milieu anglophone. De la même façon, la langue française domine maintenant, enfin, au Québec. Quelque consciente qu'elle soit du processus, la bourgeoisie francophone a clairement tiré profit de la «régionalisation» de l'économie du Québec; son contrôle de l'économie remplit une aspiration nationaliste ancienne. Le Québec ne s'est pas seulement «rattrapé» en terme de services sociaux et d'éducation, il est même devenu un modèle pour d'autres provinces canadiennes. Et le Québec est devenu une authentique société ouverte et pluraliste: lorsque les auteurs observent avec justesse que les Québécois d'origine française ont accepté la réalité multiculturelle de leur province, ils auraient pu ajouter que cette nouvelle ouverture témoigne d'un sentiment nouveau de sécurité culturelle de la part de la majorité.

Nous désirons rappeler que les faiblesses constatées ici sont insignifiantes à côté de l'énormité de la tâche accomplie par les auteurs. C'est une contribution non seulement à l'histoire du Québec mais à 
celle du Canada, ce qui est remarquable lorsqu'on considère qu'il fut conçu comme un manuel. Et si certains ont des perspectives radicalement différentes sur les événements récents de la vie politique, ceci ne réflète rien d'autre que la diversité de la réalité canadienne.

Département d'histoire

Université du Nouveau-Brunswick

BERNARD L. VIGOD

Traduction: Lise Saint-Jacques 